\title{
A New Automatic Generation Control with Heterogeneous Networks for Smart Grid
}

\author{
Zhongju Chen ${ }^{\mathrm{a}^{*}}$, Tao Li ${ }^{\mathrm{b}}$ \\ ${ }^{\text {a }}$ School of Computer Science, Yangtze University, Hubei, 434023, China \\ ${ }^{\mathrm{b}}$ School of Electronics and Information, Yangtze University, Hubei, 434023, China
}

\begin{abstract}
Current sensor can measure DC current signal for high frequency ( $\mathrm{MHz}$ magnitude), which has a very wide frequency range. And its affluent magneto resistance system can meet the needs of all kinds of electric current in the power system monitoring. In this paper, the former measure the performance levels of the network through objective point of view, the latter base on the former to join the subjective demand of distribution communication service, and to compromise subjective and objective decision result which make the business's decision for choice of network access result more reasonable and more In favor the meet business needs of Cos.
\end{abstract}

Index Terms: Heterogeneous Networks, Automatic Generation Control, GMR current sensor.

(C) 2014 Published by MECS Publisher. Selection and/or peer review under responsibility of the Research Association of Modern Education and Computer Science.

\section{Introduction}

GMR current sensor has a broad application prospect in regard to the distributed measurement on transmission line or distribution line. Compared with the traditional electromagnetic current transformer, it has the advantages which could measure high frequency ( $\mathrm{MHz}$ magnitude) DC current signal, wide measure range, high sensitivity and small size. Especially GMR can measure DC current which is extremely advantageous for the monitoring of HVDC converter station in DC transmission system [1].Compared with Hall element, it has the smaller size, the higher sensitivity, and better temperature stability, which is able to adapt to the drastic changes in the power grid environment. Compared with the new type of optical fiber current sensor, it has simple structure, simple productive process and low cost which is easy to large-scale promotion [2]. The above advantages of GMR current sensor are suitable for distributed measurement, data acquisition of smart power grid, distribution monitoring of all power grid work and the current under accident situation. Table. 1 shows it is realized the distributed real-time monitoring of smart power grid with advanced

* Corresponding author.

E-mail address: czj_yzu@ 163.com 
means of communication. In the smart power grid applications GMR current sensor instead of the traditional electromagnetic transformer, which is used to monitor transmission line, transformer substation, distributed power distribution network and electric current measure distributed measurement [3-5]. Through the advanced communication technology to collect signals to monitoring center and give the corresponding feedback, which realize the full power grid current real-time monitoring and scheduling.

Tab. 1 Several common current sensors

\begin{tabular}{l|l|l|l|l}
\hline Performance & $\begin{array}{l}\text { CT and Rogowski } \\
\text { coil: Current Sensor }\end{array}$ & Hall: Current Sensor & OFCT: Current Sensor & GMR: Current \\
\hline Schematics & Sensor
\end{tabular}

\section{Literature review}

The access selection and access control of heterogeneous converged network are respectively sent from the angle of the terminal and network control center and initially choose the most suitable Internet access through the terminal. The NCC embarks from the network overall performance to control and decide whether a network terminal is accepted [6]. These two ways to achieve the best use of network resources and the optimal state of terminal business.

The existing access selection and access control algorithm research generally includes the following categories[7]. The first is based on the business type of algorithm; it depends on the type of business for each 
call request to decide access selection or access control of the network. This kind of algorithm is embarks from that different wireless access technology has different characteristic, and the suitable business type also various. Therefore we choose the most effective network for users to access, which could guarantee Cos requirements. But it is also easy to cause the unbalanced load between the networks. The second type is based on the utility function and cost function [8]. This algorithms chooses the target network to optimize the allocation of resource by maximizing access utility or minimize access cost. The third type based on load. It gives uniform distribution for traffic load in heterogeneous system to balance the network load and make better use of system resources [9]. The main advantage of this algorithm is able to maintain high stability of the network through uniform load distribution. But the algorithm is usually making the network as a whole center. And it may cause user lower satisfaction [10].

The last class of algorithm using the intelligence theory to solve decision of business access networks problem [11]. The common intelligent optimization theory are fuzzy logic, fuzzy neural network, fuzzy multiple attribute decision making and genetic algorithm [12]. There are usually problems such as not fully considering the decision indicators, the identification of parameters or significance is highly subjective, complex computing and low universality in existing access options and access control algorithm. Although all these algorithms can improve the system performance to some extent, but there are still a larger optimization space. And there is still no research on the aspects of intelligent distribution network features and communications cos business requirements for access selection and network access control. Under the above background, we based on the intelligent power distribution heterogeneous converged network model and intelligent power distribution communication service cos requirements, to explore more reasonable and effective business access selection algorithm through relative information fusion theory.

\section{Computing model of heterogeneous converged network}

In the intelligent power distribution heterogeneous converged network environment culti-mode wireless communication terminal will be widely applied in the power distribution terminal level. It can ensure the terminal freely choose any type of wireless access networks according to their own business needs. At the beginning process of communication or during the process of communication, the terminal detecting current network environment in time and collect all the network's Cos parameters, which is shown in Fig. 1. At the same time the network selection control module in terminal will update Cos parameter values according to alternative network in time. Through the access selection model that established by proposed algorithm to select the best network in the current environment.

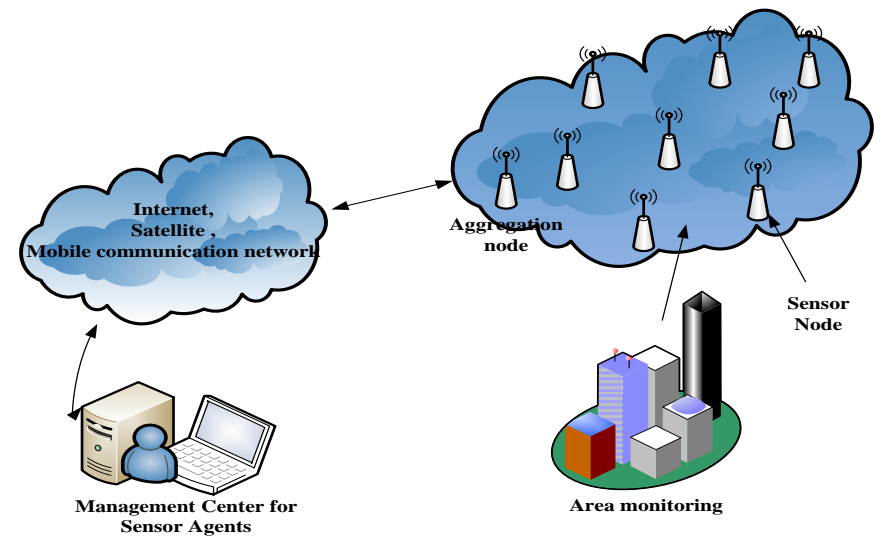

Fig.1. Framework overview 
The research approach is that the select and access of the business transformed into a typical multiple attribute decision making problems and set up multiple attribute decision model. And intelligent power distribution terminal as a decision maker, the candidate networks as an alternative solution, network performance parameters as indicators of decision making. The goal is to choose the optimal network services for the new call and switching of the power distribution communication business.

In this model, because of the different attributes the dimensional and domain space are generally different. In order to ensure its impaction fairness on decision making, we need to do standardized treatment for it at first. For cost type attributes (Such as blocking rate.etc.) it should be as small as possible. The system model described below: Standardized treatment according to Eq.1.

$$
b_{i j}=\frac{\left(\alpha_{i j}\right)_{\max }-\alpha_{i j}}{\left(\alpha_{i j}\right)_{\max }-\left(\alpha_{i j}\right)_{\min }}, i=1,2, \ldots m
$$

For efficiency of property (Such as received signal strength.etc.) it should be as big as possible. Standardized treatment according to Eq.2.

$$
b_{i j}=\frac{\alpha_{i j}-\left(\alpha_{i j}\right)_{\min }}{\left(\alpha_{i j}\right)_{\max }-\left(\alpha_{i j}\right)_{\min }}, i=1,2, \ldots m
$$

The algorithm which based on the entropy method and the Grey correlation analysis access selection can be divided into three main steps: collecting network information, processing data information and making decision. Mainly consider to collect related decision parameters is not the focus of this article in the stage of collecting each access network information. In the phase of information data treatment, the algorithm selected matrix firstly according to the strength of received signal, remaining available resources and the structure of current business blocking rate. secondly, to get the Grey correlation matrix which reflected the access network index performance levels through normative approach and Grey correlated. Finally, we employed the entropy method to calculate each performance's dimension combined with incidence matrix to get the rank of various heterogeneous network performances. According to rank result the terminal will be access to the optimum network in the stage of access decision that is target network.

For a comprehensive evaluation of the decision matrix, define the ideal network:

$$
y=\left\{\frac{\max }{i} b_{i 1}, \frac{\max }{i} b_{i 2}, \ldots, \frac{\max }{i} b_{i n}\right\}
$$

Calculate the properties of the ideal network parameters on the gray correlation coefficient:

$$
\begin{aligned}
& r_{i j}=\frac{\frac{\min }{i} \frac{\min }{j}\left|y_{j}-b_{i j}\right|+\xi \frac{\max }{i} \frac{\max }{j}\left|y_{j}-b_{i j}\right|}{\left|y_{j}-b_{i j}\right|+\frac{\max }{i} \frac{\max }{j}\left|y_{j}-b_{i j}\right|} \\
& (i=1,2, \ldots m ; j=1,2, \ldots n)
\end{aligned}
$$


Where $\xi$ is resolution factor, the value is [0,1], usually get $0.5, \frac{\min }{\mathrm{i}} \frac{\min }{\mathrm{j}}\left|\mathrm{y}_{\mathrm{j}}-\mathrm{b}_{\mathrm{ij}}\right|$ are the two smallest difference, $\frac{\max }{\mathrm{i}} \frac{\max }{\mathrm{j}}\left|\mathrm{y}_{\mathrm{j}}-\mathrm{b}_{\mathrm{ij}}\right|$ are the two maximum difference.

\section{Simulation results and analysis}

The Grey correlation analysis is essentially through comparing the geometry proximity of data sequence curves to judge the correlation degree. Generally speaking, the closer of geometric shapes, the more collection it is, and it will be less on the contrary. By calculating the reference sequence and compare sequence of Grey correlation degree, we analysis the advantages and achieve the optimal and worse order of the evaluation objects. This method makes up for the defects of system analysis by using mathematical statistical method.

It is apply to various sample size rule and existent and non-existent sample rule, And it has small amount of calculation. We employed the GRA in heterogeneous network access selection algorithm. First of all, we according to the performance parameters of the real-time heterogeneous system level to define an ideal network. Then calculate the Grey correlation degree of available network and the ideal network. Finally calculate each available network comprehensive evaluation value and rank comprehensive evaluation value then we can determine the optimal target network.

In order to validate the proposed the E - GRA algorithm the performance, we make simulate compare analysis of the MLB algorithm from literature with E - GRA algorithm on network performance. Simulation model is Limax and TD- LTE two overlapping coverage of heterogeneous wireless network scenarios. In the process of simulation, randomly generated initial business distribution of the network and 60 new power distribution communication business. The type and occupied resources refer to Fig.2, which shown the randomly generated business types and are randomly distributed in the overlapping coverage area.

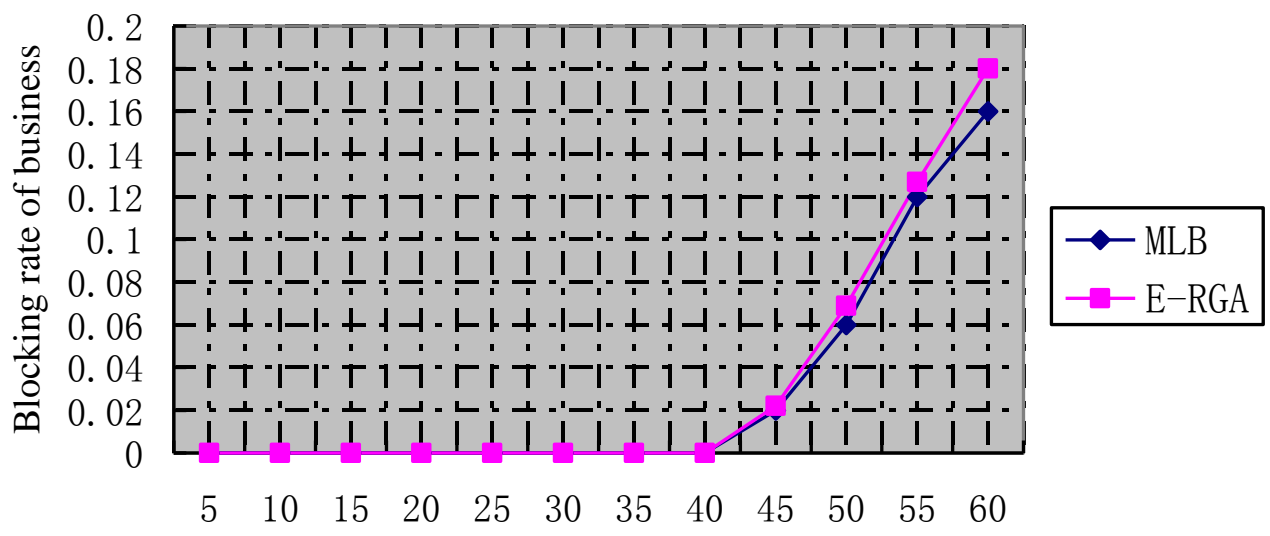

The number of users

Fig.2. Average service blocking probability

In Fig. 2 show, when traffic is less than 48 distribution, because the network has sufficient resources to accept service, service blocking rate under the two algorithms are 0; With increasing number of businesses, business blocking rate gradually increased. 


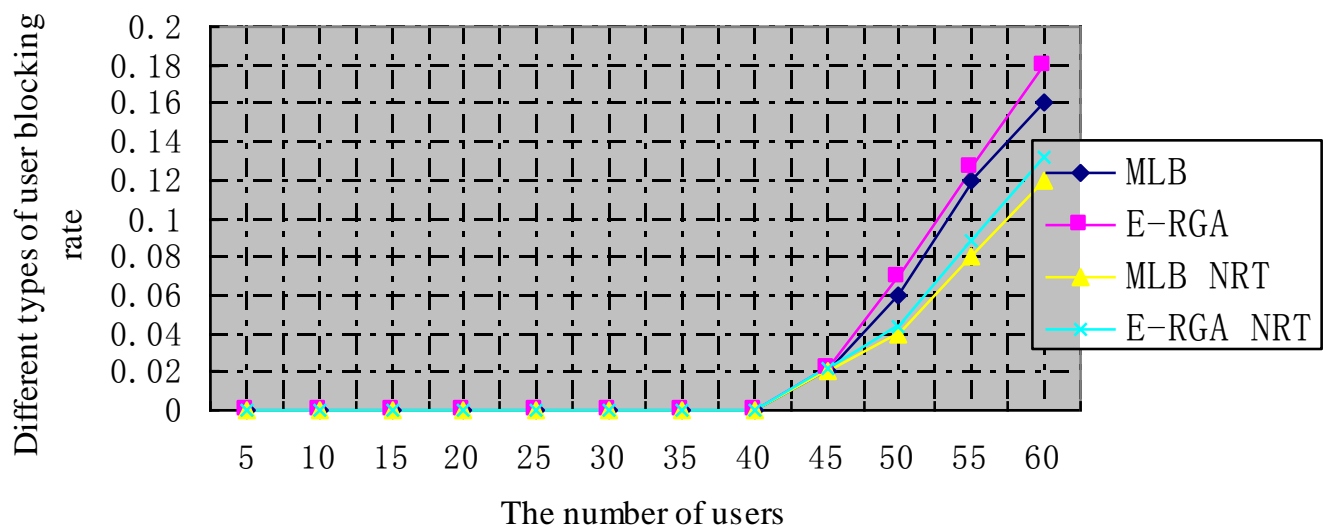

Fig.3. Blocking probability of different services

In Fig. 3 show, In both algorithms in real time and non-real time services were average blocking rate. As can be seen, the number of users increases, the proposed two businesses mean blocking rate E-GRA algorithm algorithms are always more than MLB average blocking rate.

\section{Conclusion}

The business of access and selection strategy and network control access strategy in internet access in heterogeneous converged network is an important research content in the wireless resource management. In the view of the existing algorithm of judgment index considering is insufficient, parameters or dimension has strong subjectivity, calculation is complex and low generality problems, we proposed the access selection algorithm respectively based on the entropy weight method and the Grey correlation analysis, and based on the rough set theory and analytic hierarchy process.

It is worth to point out that based on the performance simulation and analysis of the algorithms can shown the proposed two algorithms has obvious effect to implement the network load balance between each network. That is to say rationally employ the resources in heterogeneous converged networks and control and scheduling for the business access request which is an effective way to realize heterogeneous network load balance. The subsequent chapters will began to focus on the discussion of load balance algorithm in heterogeneous converged network resource management.

\section{Acknowledgements}

Project supported by the National Natural Science Foundation of China (No. 60973012) and Technology Development Project of Jingzhou (No. 2012CA20).

\section{References}

[1] Pantazis N A, Vergados D D. A survey on power control issues in wireless sensor networks[J]. Communications Surveys \& Tutorials, IEEE, 2007,9(4):86-107. 
[2] Bhatti S, Xu J. Survey of target tracking protocols using wireless sensor network: Wireless and Mobile Communications, 2009. ICWMC’09. Fifth International Cor^erence on, 2009[C]. IEEE.

[3] NIST Framework and Roadmap for Smart Grid Interoperability Standards Release 1.0[S].

[4] B. Lu, T. G. Habetler, R. G. Harley, et al. Energy evaluation goes wireless [J], IEEE Industry Applications Magazine, 2007, 13(2): 17-23.

[5] G ran N. Ericsson. Cyber security and power system communication-essential parts of a Smart Grid Infrastructure [J]. IEEE Transactions on Power Delivery, 2010, 25(3): 1501-1507.

[6] Save Stanic, Suresh Subramaniam, Gokhan Sahin. Active monitoring and alarm management for fault localization in transparent all-optical networks [J]. IEEE Transactions on Network and Service Management, 2010, 7(12): 118-131.

[7] Luo Jinran, Huang Shanguo, Zhang Jie, et al. A novel multi-fault localization mechanism in PCE_based multi-domain large capacity optical transport networks[C]//OFC/NFOEC Optical Fiber Communication Conference and Exhibition/National Fiber Optic Engineers Conference . Los Angeles, CA, USA: the Optical Society of America (OSA), 2012.

[8] Ali Mohammed L, Pin-Han Ho, Tapolocai J, et al. M-burst: a framework of SRLG failure localization in all-optical networks[J] . IEEE/OSA Journal of Optical Communications and networking, 2012, 4(8): 628-638.

[9] Novosel D. Emerging Technologies in Support of Smart grid[c]. IEEE Power and Energy Society General Meeting - Conversion and Delivery of Electrical Energy in the 21st Century. Pittsburgh, Pennsylvania, USA, 2008.

[10] The National Energy Technology laboratory. A Systems View ofthe Modern Grid[R / OL]. [2007 01 - 22]. http: / / ww. neff. doe. gov / mordemgrid.

[11] The National Energy Technology Laboratory. The modern Grid Initiative. Pittsburgh. PA. USA: NETL, 2007.

[12] F.J. Molina, et al., Automated Meter Reading and SCADA Application for Wireless Sensor Network[C], Proceeding of ADHOC-NOW, New York: IEEE Press, 2003:223-234.

\section{Author(s) Profile}

Zhongju Chen graduated in computer science from Jianghan Petroleum College, China, in 1998, and received the M.S. in Geo Probe and information technology from Yangtze University, Hubei, China, in 2004. $\mathrm{He}$ is currently working toward the Ph.D. degree in Computer systems and structures at Wuhan University, Hubei, China. His research interests include Computer Network, Database Application, System Structure and Artificial Intelligence.

How to cite this paper: Zhongju Chen, Tao $\mathrm{Li}$,"A New Automatic Generation Control with Heterogeneous Networks for Smart Grid", IJEM, vol.4, no.1, pp.25-31, 2014.DOI: 10.5815/ijem.2014.01.04 